\title{
Analysis of the reasonableness of the current level of required accuracy of satellite navigation of consumer systems civil mobile means
}

\author{
Lev N. Lysenko, Vsevolod V. Koryanov, and Alexey G. Toporkov \\ Bauman Moscow State Technical University, 105005 2-ya Baumanskaya ul. 5, Moscow, Russian Federation
}

\begin{abstract}
Analysed the paper presents the analysis of the essential accuracy level expected from the modern fundamental coordinate and time data of both existing and prospective satellite navigation and telecommunication systems, while solving the real-world problems of ballistic and navigational support. The paper shows that the respective analysis of the effect of the navigational coordinate and time data uncertainty should be performed based on the estimates of the required ballistic and navigational support of the satellite systems, taking into account the differences between the theoretically attainable level and the practically feasible level of the accuracy for the fundamental coordinate and time data
\end{abstract}

\section{Introduction}

Determining essential elements of the modern coordinatetime and navigation support (CTNS) is created and successfully operated in the Russian Federation global navigation satellite system (GLONASS).

As a consequence, modernity has emerged selfsufficient integrated system that provides users the ability to create an independent specialized consumer system (CS), able to solve the problem of CTNS in the specific conditions of operation with a given level of reliability and accuracy.

Basic determinant of the achievement of CS limit capabilities is the level of accuracy of the fundamental coordinate and time support (FCTS), without which it is impossible to create a satisfying private demands CS [1].

The interaction between FCTS and CTNS is clear: the level of CTNS is uniquely determined by the achieved level of development of FCTS, on the other hand highly uncertain due to the lack of correctly and reliably limits the accuracy of demand characteristics and parameters of FCTS and especially the impossibility of strict accounting, the level of uncertainty in the ballistic and navigation support (BNS) satellite navigation systems (SNS).

In this regard, note that the key performance indicators of CTNS CS, integrally defined locating accuracy, availability, integrity and continuity of navigation field, may not be directly linked to numerical precision the values of the parameters of FCTS.

At the same time, it is clear that the new level of requirements to CTNS not possible without further research in the field of FCTS. These studies are very diverse and extremely complex and unique measuring tools [2]. The exploration and development of the corresponding field of knowledge is a time-consuming, great effort and considerable material resources, which makes it relevant to the assessment of justification of requirements to the level of required accuracy of navigation definitions on the basis of radio-navigation satellite systems (RNSS).

\section{The concept and indicators of the accuracy of navigation satellite definitions}

Ensuring operational ephemeris (OE) navigation satellites (NS) includes the determination and prediction of orbits in the inertial parameters of the CS, the calculation of the Earth rotation parameters (ERP) and the recalculation of parameters of motion of the satellite with the use of ERP in the non-inertial coordinate system (CS) current version (characterized by the existing level of uncertainty of the parameters of FCTS), update as often as necessary, these data on Board NS for frames of digital information in the navigation signals, including parameters of the almanac.

Calculation of ephemerides and their accuracy factor is based on the results of measurements of the distance call-outs and/or pseudo-range of no-request systems, and, in some cases, data of Very Long Baseline Interferometry (VLBI).

The orbits are specified on the daily plan for each stage, and measuring the interval refinement of ephemeris corresponds to the circuit of the satellite tracks overlap. In systems of high-accuracy determination of ephemeris and time corrections in the interests of ephemeris-time support processing only measurements of no-request systems global network. They also calculate long-term (90 days) forecast of the state almanac and operational 
frames of digital information for up to 10 days to maintain the assisting technologies.

Usually considered that the error actually determine the ephemeris from the standard accuracy is about 2-3 times lower than the errors caused by errors in assigning frequency-time parameters.

Operational frequency-time support implements the synchronization of on board clock relative to the time scale of the system by calculating and predicting the onBoard time scale relatively time scale of the system update on Board Time-Frequency Corrections (TFS) placed in the navigation frame.

To predict Board time scale $8 . .12$ hours is used the two-day array of results, which is approximated by a linear polynomial on the interval of extrapolation.

When you upgrade the TFS at every turn of the standard deviation (SD) errors in the mutual synchronization of any two Board time scale of navigation satellite is approximately from 5 to $8 \mathrm{~ns}$, which introduces error in the determination of the location of the consumer at the rate of 2.0 to $2.8 \mathrm{~m}$ (2 SD), and to ensure the TFS equal precision data for all satellites of the system in principle is not possible. At the present level differences in accuracy between the TFS "best" and "worst" navigation satellite can exceed 5-6 times.

It would seem that the above considerations can serve as a quite distinct grounds for unequivocal determination of the precise characteristics of the domestic GLONASS and American GPS (Global Positioning System) and map them.

Usually given in the literature data should be considered as rather arbitrary and approximate. The case is as follows.

The accuracy of navigation definitions has significant impact on the location of the satellite in the constellation relative to the user. For planning navigation support in different parts of the Earth it is necessary to have cards of equal accuracy, which should be marked with lines of equal values of Position Dilution Of Precision (PDOP). Therefore, for global satellite navigation system can only talk about the potential accuracy of navigation definitions, which together form "field precision of the satellite navigation system ". When this field precision varies with time due to the motion of the satellite relative to the Earth's surface and variations of the configuration of the selected constellation.

If the structure of the satellite navigation system regular, i.e. it repeats itself after a certain period of time, the precision can be allocated to the elementary structural zone, which is repeated in time and space. For the prototype GPS system "Navstar", for example, the size of such a zone area was 1.5 hours time and 60 degrees in longitude. In the period under consideration (early-mid 80 -ies of the last century) standard deviation determine the location of the object using said system, respectively $7 \ldots 25 \mathrm{~m}$, height $10 \ldots 43 \mathrm{~m}$, the maximum measurement error of radial velocity was $0.06 \mathrm{~m} / \mathrm{s}$.

A comparison of the level of navigation accuracy for different satellite systems is possible only when there is at least similar prerequisites for achieving the same goal, which is not always taken into account. As a consequence, we compare the accuracy characteristics of GLONASS with GPS in the structure of the global system of WAAS (Wide Area Augmentation System) differential navigation (GDGPS), utilizing up to 30 stations in redundant form, or at least 12 stations that are guaranteed to be optimally located throughout the globe.

With all the conventions of existing assessments though objectively it is possible to consider [3] that at low angles of shading (at the angle designated signals up to $5^{\circ}$ ) orbital group (OG) GLONASS-24, providing 100 percent availability of navigation except for small areas at latitudes $\pm 28^{\circ} \ldots \pm 30^{\circ}$, the accuracy is inferior to GPS on almost $20 \%$; at high angles of shading (elevation $\geq 25^{\circ}$, which corresponds to the urban and mountainous conditions) OG GLONASS-24 1.5-2 times lower than on the global availability of not only GPS but also GALILEO satellite navigation system-27 (European Union) and COMPASS (China).

Leaving aside the problems of accessibility, let us state the assumption that the existing backlog on navigation accuracy from GPS (USA) was the reason for the language requirements of the current stage of development of GLONASS aimed at achieving a precision navigation definitions up to $0.1 \ldots 0.6 \mathrm{~m}$ (SD), which significantly exceeds the guaranteed accuracy of operating system.

\section{Assessment of demand indicators of present level of accuracy of satellite navigation.}

The achievement of a previously specified level of accuracy of navigation definitions put forward, according to numerous statements of representatives of the Institute of applied astronomy of Russian Academy of Sciences (RAS), the accuracy requirement is fundamental segment $\mathrm{KBO}$ at the level of about $3 \mathrm{~cm}$ in determining the position of the center of the geoid (the Geocenter) with an error less than $1 \mathrm{~cm}$, ground $\mathrm{CS}-5 \cdot 10^{-10}(3 \mathrm{~mm}$ coordinates), and in the sky CS - location accuracy should characterize the level of $0.02 \mathrm{~ms}$ for the arc. of the standard ICRF and $0.1 \mathrm{~ms}$ of the arc. for geodetic sources.

To understand the reachability of such requirements, we note that they cannot be guaranteed without the installation of beacons for VLBI observations at the poles of the moon, determining the ephemerides of Solar system bodies at the level of $10^{-11}$, the daily non-stability frequency standards and time of $10^{-16}$ to $5 \cdot 10^{-17}$ with finally, the daily forecast of the orbits of GLONASS satellites with a precision better than $1 \mathrm{~mm}$. About the unfeasibility, at least, the latter condition is, in principle, cannot speak.

But if so, the question naturally arises about the practical validity of the formulated requirements to guarantee the level of accuracy of FCTS.

Moreover, we note that the analysis of the impact of uncertainty, knowledge of the parameters of CTNS for RNSS on the basis of the accuracy of their required BNS need to be implemented taking into account the differences between the theoretically achievable level of accuracy of parameters of FCTS and their practical level 
of precision determined by the requirements of specific consumers.

So, there are two aspects to answer the question posed.

1. Are there any alternatives to improving the accuracy of FCTS to achieve the desired level of accuracy of navigation definitions of consumers?

2. What is the intrinsic demand of modern level of accuracy of satellite navigation, at least from the major consumer of civilian systems?

For a better understanding of the substance of the first part of the matter under discussion will limit ourselves to the analysis of problems BNS geostationary satellites. No matter how accurately we have not defined the parameters of FCTS, the absolute accuracy of their knowledge is impossible to achieve and therefore almost impossible to enforce the actual conditions of stationarity.

Objectively, uncertainties knowledge of factors such as off-center gravity of Earth, the inaccuracy of the knowledge of attraction of the Sun, moon, Venus, Jupiter and the other inner planets of the Solar system, direct and reflected radiation pressure, tidal deformation of the Earth, the ocean tides, the corrections General relativity and other factors, will inevitably lead to a shift (drift) of the satellite relative to the earth.

Despite the fact that the acceleration experienced from, for example, a direct light pressure (level $10^{-7} \mathrm{~m} / \mathrm{s}^{2}$ ) and tidal deformations of the Earth $\left(10^{-10} \mathrm{~m} / \mathrm{s}^{2}\right)$ are, at first glance, almost intangible value, after 30 days the resulting maximum deviation from the nominal setups will be respectively about 2.4 and $8.5 \mathrm{~m}$.

As a consequence, suggest the following possible solutions to the problem: either to increase the accuracy and reliability of knowledge of the parameters of FCTS as possible, and technically to implement precision BNS, either consciously (taking into account the existing possibilities of technical systems) limit the accuracy of BNS to the level of allowed deviation caused by drift, keeping the parameters of the orbit closest to the ideal stationary values in the neighborhood of a given point of standing through the management of the movement of the center of mass of geostationary satellites, which consists in the periodic correction of its orbit.

The first generation displayed on geostationary Earth orbit (GSO) satellites is adjusted, as a rule, only the parameters that define the offset of the satellite in longitude to the satellite is guaranteed to remain within "geostationary belt" ("Clarke belt").

Since that time much has changed. Used in past classical methods for determining the ephemeris and, as a consequence, astronomical constants of the moon and planets were based mainly on optical observations. Revolutionary was the widespread use of radio and especially laser astronomical measurements. Thanks to this data situation, at least for the inner planets has drastically changed. Accumulation of measurement results obtained using the AMC (mainly Viking, Pathfinder, MGS and Odyssey), had developed highprecision theory of the ephemerides of the inner planets with a precision of milliseconds of arc. The standard deviation of the observations about estimated values for mercury is $1.4 \mathrm{~km}$ for Venus and Mars $-0,7 \mathrm{~km}$, for
AMC Viking - $8.8 \mathrm{~m}$, Pathfinder - $5.1 \mathrm{~m}$, MGS and Odyssey $-1.4 \mathrm{~m}$.

As a result, managed to create a dynamic model of high-precision ephemerides of planets and the moon (EPM). The best of existing foreign models of DE405/LE405 in this case exceeds, for example, the previously used model DE200/LE200 by the accuracy of assigning astronomical unit (a.u.) length (a.u. = $149597870696,3 ; 0,1)$ is several orders of magnitude. Developed the Institute of Applied Astronomy RAS model are comparable in accuracy with the specified DE405/LE405. Domestic dynamic model EPM2004 (Pitjeva, 2004) takes into account mutual perturbations of the major planets and the moon, compression of the Sun, the effects of physical libration of the moon, perturbations from the 301 biggest asteroids and the massive asteroid ring located in the plane of the Ecliptic. Ephemeris converted to the ICRF system and agreed with the resolution - mi XXIII and XXIV General assemblies of the International astronomical Union.

In relation to the refinement of geodetic parameters of the Earth to Refine the resonant harmonics of the gravity of Earth, primarily in the interests of GPS, in the middle of 1980 in the U.S. program was implemented COGOES (international program of optical observations of geo stationary satellites).

Note, incidentally, that in the USSR at that period there were no conditions for the implementation of geodetic and geophysical softwares required level, due to the lack of national special spacecraft, similar to that used in the USA to improve the accuracy of ephemeris support navigation satellite systems such as GPS.

This, in turn, has improved the level of accuracy BNS excretion geostationary satellites to officially declare a point of standing in GSO, as well as to find an acceptable compromise on the size of the dead zone of permissible deviations of the satellite from the nominal value, defining the value of energy losses to the correction of the orbit on the one hand, and, on the other, restrictions on the minimum allowable angular distance between adjacent satellites in GSO, set by international agreements, reasons of electromagnetic compatibility of satellite radio. The relevant regulating document serves as "the radio Regulations" / Edition of 2004. No. 119-04REV-04, imposing the restriction of $\pm 0.1 \circ$ in the recent past, adjusted up to $\pm 0.05^{\circ}$ currently, for the deviation of the satellite in longitude relative to the nominal setup [1].

In order to perform the required corrective maneuvers hold the satellite at a given longitude and latitude with minimal energy consumption and with a sufficient precision, needed to have the most accurate information about the parameters of the real orbits. The navigation process is based on Measurements of Current Navigation Parameters (MCNP) using special measurement tools and statistical processing of results of measurements using the most rational of mathematical models and computational schemes that are part of BNS utilization.

While for most practical applications geostationary satellites, in principle, it is sufficient to account for the influence of the following perturbation: 
- the force components of the gravity of Earth with the deduction of harmonics of the spherical functions of the geopotential, not above the 8 th order (with the fame para - meters 36 harmonic model WG84 and 70 for model EGM96);

- the gravitational attraction of the Sun and moon as point masses;

- direct light pressure;

- implementation errors of the thrust vector of the propulsion unit (module and orientation).

Data from the generalized requirements of most CS CTNS follows that none of the consumer systems does not imply the need reaches the level of the average values of SD centimeter accuracy for solving problems of navigation definitions of satellite navigation systems.

As an exception can be noted only marginal (maximum) accuracy levels the accuracy of determining when approaching and landing aircraft according of the International Civil Aviation Organization categories (max $0.2 \mathrm{~m}$, min $2 \mathrm{~m}$ ) and prospective levels of precision maneuvering and conducting the specialized works in the water area of bodies of water and ports (max $0.1 \mathrm{~m}$ min $3.5 \mathrm{~m}$ ). We emphasize again that we are talking about extreme levels of precision, which in most cases is not common to use in design development.

Individual attention requirements of substation surveying software and high-precision monitoring of displacements of engineering structures difficult.

Note that for the solution of applied problems of geodesy measurements performed on points of the geodetic control network using the methods of relative definitions. State geodetic network in this case is based not only on satellite geodetic network of 1 class, but also on a fundamental astronomic-geodetic network, as well as high-precision geodetic network. In this system besides written long existing and widely used network triangulation and polygonometry different classes. Everything else for reading surface, in particular in the CS-95, adopted not the true surface of the Earth, and the surface reference ellipsoid of Krasovsky.

In this setting the output centimeter level of accuracy of astronomical and geophysical networks in combination with new high precision points of the satellite network of permanent differential stations, of course, is not an unsolvable problem. Moreover it should be considered real and millimeter level, relevant to the solution of some problems of geodynamics (earthquake prediction in seismically active areas). As declared millimeter level accuracy for cadastral surveying and fixing the boundaries of land use, such claims cannot be taken seriously. Here, at least, desirable (but not substantiated) is valid.

This gives grounds to assume that from the point of view of the practical demands of the consumers of satellite navigation, at least for civil purposes, the existing requirements for the level of accuracy of the means for FCTS is overstated [1].

As to the provision of the required level of accuracy of even the "precision" of space objects, as you know, updated currently means Ground Computer-Aided Control Complex (GCACC) allow or will allow in the near future to carry out the measurement of the orbit parameters of spacecraft in the altitude range $200 \ldots 40$ $000 \mathrm{~km}$ with an accuracy (maximum error) in the range of $1 \ldots 30 \mathrm{~m}$ and the radial velocity of $0.001 \ldots 0,003 \mathrm{~m} / \mathrm{s}$. Such accuracy is quite sufficient for high-precision navigation definitions most of the existing types of spacecraft, in particular, for example, to keep the type of communications satellites "Express-A" in the permissible range is $\pm 0.05^{\circ}$, implying the need to define the parameters of their orbits at: 0,9 arc. minute - latitude of sub-satellite point; 0,3 arc. minute - longitude of subsatellite point; 0.15 - period of satellite revolution.

The satisfaction of increased demands BNS advanced space funds can only be secured through the involvement of satellite navigation equipment.

\section{Conclusions}

1. The level of requirements for navigation software from various categories of consumers, gives reason to conclude that none of the existing substation CS CTNS not imply the need reaches the level of the average values of SD centimeter accuracy for solving problems of navigation definitions of satellite navigation systems.

In this regard, requirements to the complex of FCTS on the level of knowledge of the position of the Geocenter with error not exceeding $1 \mathrm{~cm}$, ground CS $5 \cdot 10^{-10}$ ( $3 \mathrm{~mm}$ for coordinates), the celestial CS $-0.02 \mathrm{~ms}$ for the arc of the standard ICRF and $0.1 \mathrm{~ms}$ of the arc. for geodetic radio sources, is overstated.

2. Given that the achievement of the stated values focus on the need to ensure in principle unattainable daily predict the orbits of GLONASS satellites with a precision better than $1 \mathrm{~mm}$, these requirements appear to be superfluous not only for the state of the art, but also in relation to the long term.

3. Navigation errors are not only caused by errors of determination of orbit parameters according to the processing results of MCNP, but also the forecast errors due to inaccurate descriptions of the forces at work, including errors in the execution of maneuvers.

Not accounting for this circumstance is caused first of all by those considerations that the maximum interval of forecast ephemeris and TFS used in the ephemeris-time support system is always less than a predetermined interval of "aging", which, as is generally assumed, can always be reduced if it is used, the value begins to have a noticeable impact on the precision technical characteristics of ephemeris-time support system.

Currently, the interval of "aging" ephemeris-time information in the GLONASS system for normal functioning period of the system is $12 \ldots 14$ hours. Thus, it is common to assume that the semidiurnal forecast ephemeris and TFS for almost MCNP should not introduce errors, or they are so small that they can be neglected. In fact, everything is determined by the required level of accuracy of navigation definitions. For today's "meter-level" errors semidiurnal forecast can be neglected, but the exit to "the centimeter level" has radically changed the situation. Reducing the interval of "aging", which is widely accepted to refer as an 
"elementary solutions" is not unlimited and cannot serve as a guarantee for the solution.

4. In order to provide a predetermined sufficiently small value of the range of aging ephemeris-time information is necessary to measure network keys, which help to clarify the ephemeris and TFS, would not break, required large intervals of "aging" and that the topology of filling of the measuring stations the GCACC would ensure the bookmarks with the corresponding period. The current network of filling GCACC allows you to increase the frequency of bookmarks by no more than 1-2 hours, which is insufficient for achieving a promising level of accuracy of satellite navigation.

\section{References}

1. Lysenko L.N., Koryanov V.V., Toporkov A.G. Evaluation of satellite navigation accuracy requirements based on the analysis of the current state of commercial coordinate and time navigation support systems, Vestnik BMSTU, Mech. Eng, 47 (2015).

2. Finkelstein A.M., Ipatov A.V., Skurikhina E.A. The fundamental coordinate and time support for the GLONASS system means RSDB-network QuasarKVO. Proc. of IAA RAS, 77, 608 (2007).

3. Stupak G.G., Lysenko L.N., Betanov V.V. Status and Perspective for Improving Orbital Structures of Navigation Satellite Systems, Vestnik BMSTU, Priborostr, 3 (2014).

4. Lysenko L.N., Betanov V.V., Zvyagin F.V. Theoretical Foundations of ballistics Navigation Support Space Missionsw, 518 (2014).

5. Urlichich Yu.M., Yezhov S.A., Zhodzishsky A.I., Kruglov A.V., Makhnenko Yu.Yu, Modern technology navigation geostationary satellites, 271 (2006). 